\title{
Raíces Dentales Humanas Normales y con Perlas de Cemento. Comparación Histológica de Estructuras
}

\author{
Normal Human Dental Roots and with Cementum Pearls. \\ Structural Histological Comparison
}

\author{
Alicia Kohli*; Stella M. Pezzotto** \& Leonor Poletto**
}

KOHLI, A.; PEZZOTTO, S. M. \& POLETTO, L. Raíces dentales humanas normales y con perlas de cemento. Comparación histológica de estructuras. Int. J. Morphol., 31(3):1020-1025, 2013.

RESUMEN: Durante la vida del diente en boca se deposita cemento celular en el ápice a fin de compensar el desgaste oclusal considerado normal. Al extraer un diente, en raras ocasiones es posible visualizar excesos de cemento con forma de perlas bien delimitados y duros al tacto, ubicados en otro sector radicular diferente al ápice. Nuestro objetivo fue comparar estructuras en ambos tipos de cemento a fin de hallar diferencias histológicas. Se utilizaron 20 dientes permanentes con perlas (G1) y 20 con raíces normales (G2). Las raíces de G1 se cortaron con disco metálico de grano fino a baja velocidad en sentido transversal, a la altura de la parte más prominente de la protuberancia, las raíces de G2 se marcaron en tercios y se cortaron en sentido transversal aproximadamente a la altura de la mitad del tercio apical y de ambos grupos se obtuvieron un segmento que fue reservado y otro que fue preparado con técnica por desgaste para visualización con MO a menor y mayor aumentos. Los resultados se compararon con prueba t de student, las variables categóricas se compararon con pruebas de Fisher significación 5\%. Se obtuvieron 40 dientes de pacientes adultos, 42,5\% masculinos y 57,5\% femeninos, edad promedio $61 \pm 16$ en G1 y $55 \pm 18$ en G2 (p=0,289). El contorno exterior de las perlas fue liso, conservando su perímetro, la zona granular de Tomes fue visible al igual que las lagunas, que fueron menos abundantes, de mayor tamaño y con distribución desordenada llegando en menor proporción al borde del tejido comparado con cemento normal.

PALABRAS CLAVE: Cemento dental; Hipercementosis; Perlas de cemento.

\section{INTRODUCCIÓN}

Durante la vida del diente en la cavidad oral, se deposita cemento celular en el ápice a fin de compensar el desgaste oclusal, manteniendo los dientes en contacto (Avery \& Chiego, 2007). Su aspecto histológico puede ser de capas de cemento dispuestas en láminas ordenadas o irregulares con abundantes cementocitos, líneas de reposo oblicuas o curvadas con respecto al eje mayor de la raíz, con vasos incluidos o bien tener el aspecto de los sistemas de Haver del tejido óseo compacto. Un tipo de hipercementosis, más difícil de observar, es la difusa que se puede presentar con formas ovoide o esférica y estar ubicada en tercios medio y cervical de la raíz. Esta provoca una deformación definida, histológicamente semejante a la apical (Cabrini, 1980; Abramovich, 1999).

El tejido cemento presenta células o cementocitos ubicados dentro de una cavidad de la matriz mineralizada llamada cementoplasto o laguna los que se distinguen fácil- mente en los preparados por desgaste. Esta célula oval, con su eje mayor paralelo al eje mayor del diente, es una célula semiatrófica o en estado de reposo, tiene su actividad reducida para sintetizar colágeno aunque puede elaborar sustancia fundamental amorfa.

La histología de la hipercementosis observada con tinción metálica reveló alteraciones microscópicas como diferentes formas y tamaños de los cementocitos y la fragmentación de sus procesos citoplasmáticos (Lia et al., 1978). Un estudio con microscopio confocal sugirió que los cementocitos podrían tener un rol en la calcificación del cemento secundario (Kagayama et al., 1997).

Al extraer un diente, en raras ocasiones es posible visualizar excesos de cemento con forma de perlas bien delimitados y duros al tacto, ubicados en otro sector radicular diferente al ápice. Estas protuberancias podrían estar rela-

\footnotetext{
* Facultad de Odontología, Universidad Nacional de Rosario, Rosario, Argentina.

** Consejo de Investigaciones, Universidad Nacional de Rosario, Rosario, Argentina.
} 
cionadas con procesos inflamatorios crónicos, con la progresión de enfermedad periodontal y con mala nutrición (Corruccini et al., 1987; Aguiló \& Gandía, 1995). El objetivo de este trabajo fue comparar las características histológicas de hipercementosis apicales con depósitos de cemento no apical en estas protuberancias.

\section{MATERIAL Y MÉTODO}

Se utilizaron 20 dientes permanentes extraídos, superiores e inferiores, en cuyas raíces se observaron depósitos de cemento alejados del ápice radicular, y otros 20 dientes de forma radicular considerada normal, de adultos de ambos sexos, $42,5 \%$ hombres y $57,5 \%$ mujeres, con una edad promedio de 61 años (16-79). Todas los dientes utilizados fueron extraídos por indicación, y con el consentimiento firmado de los pacientes, quienes conocieron de su inclusión en un trabajo de investigación.

Las raíces con evaginaciones, G1, se cortaron con disco metálico de grano fino a baja velocidad, también en sentido transversal, a la altura de la parte más prominente de la protuberancia, obteniéndose un segmento que fue reservado y otro que fue preparado con técnica por desgaste para observar la fase inorgánica con microscopio óptico (MO) a menor (10X) y mayor aumento (45X). Las raíces normales, G2, se marcaron en tercios y se cortaron en sentido transversal aproximadamente a la altura de la mitad del tercio apical con el objetivo de obtener un segmento de tejido. Los segmentos de G1 y G2 se prepararon con técnica por desgaste y los otros segmentos fueron reservados a fin de realizar un estudio posterior. Se desgastó el tejido duro con el uso de piedras de grano grueso, mediano y fino hasta obtener una lámina transparente la que fue adherida al portaobjeto con bálsamo de Canadá y protegida con cubreobjeto.

Se calculó para cada grupo edad promedio y desvío estándar, y se compararon mediante una prueba t de student. Para las variables categóricas se calcularon proporciones, las que se compararon por medio de pruebas de Fisher. Se trabajó con un nivel de significación del 5\%.

\section{RESULTADOS}

Los dientes fueron separados en 2 grupos a fin de comparar las características histológicas. Veinte dientes con depósitos anormales de cemento visualizados después de realizada la extracción (G1) y veinte presentaron raíces con- sideradas normales (G2), Figura 1. La edad promedio de G1 fue $61 \pm 16$ (24-79 años) y de G2 de 55 \pm 18 (16-78 años), sin diferencia $(\mathrm{p}=0,289)$.

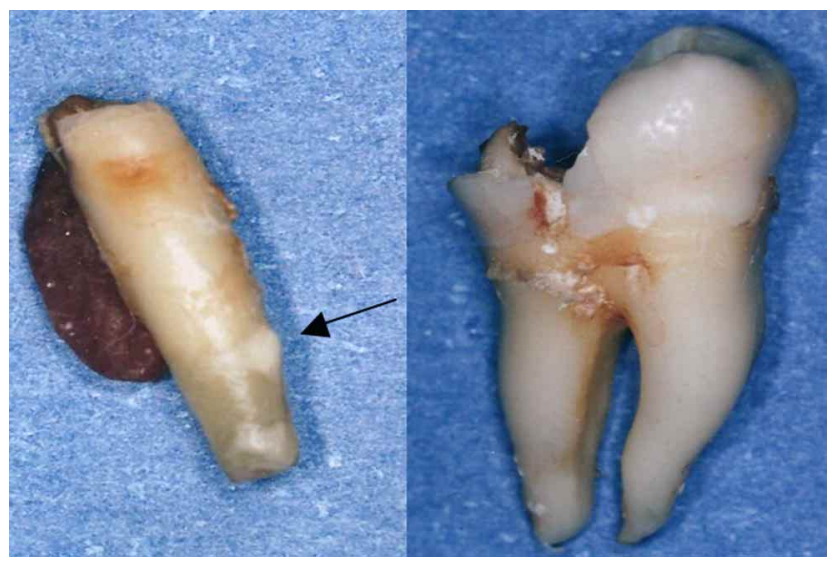

Fig. 1. Raíz con perla (flecha) y raíz normal, respectivamente.

El análisis histológico del desgaste a 10X mostró predominio del color marrón del cemento en ambos grupos. El ancho de cemento en G1 fue mayor en el sitio de la lesión diferenciándose del resto de la raíz; en G2 el espesor fue homogéneo y fino en toda su extensión ( $\mathrm{p}=0,001)$.

Al desgaste, el perímetro de la perla se conservó en el $85 \%$, mientras que en las raíces normales el perímetro del cemento radicular lo fue en el $30 \%(\mathrm{p}=0,001)$.

La zona granulosa de Tomes, que se encuentra separando los tejidos cemento y dentina, fue visible en el $83 \%$ del G1 y en el $63 \%$ del G2 (p=0,004). Esta se visualizó en forma completa en el $85 \%$ de las lesiones, en las raíces normales fue completa en el total de los casos.

Las lagunas fueron visibles en la totalidad de las perlas y no lo fueron en el $90 \%$ del G2 ( $<<0,001$ ); fueron abundantes en el 50\% del-G1 y en el $80 \%$ del -G2 (p=0,008). En las perlas se observaron lagunas agrupadas; en las raíces normales éstas fueron escasas (Fig. 2).

En la Figura 3 se muestra la cantidad de lagunas, su visibilidad relacionada con la zona granulosa de Tomes y la conservación del perímetro de las raíces en ambos grupos.

En las perlas la distribución de lagunas no siempre mostró su eje mayor paralelo al eje longitudinal de la raíz, en algunas presentaron diversas posiciones, incluso lo fueron perpendiculares a dicho eje, por cuyo motivo se las calificó como desordenadas (Fig. 4). Esta disposición se encontró en el 89\% de las perlas y en el 10\% del G2 (p<0,001); se las halló hasta el borde del tejido sólo en un 33,3\% del G1 y en un $60 \%$ del -G2 ( $\mathrm{p}=0,121)$, ambas características se señalan en la Figura 5. 


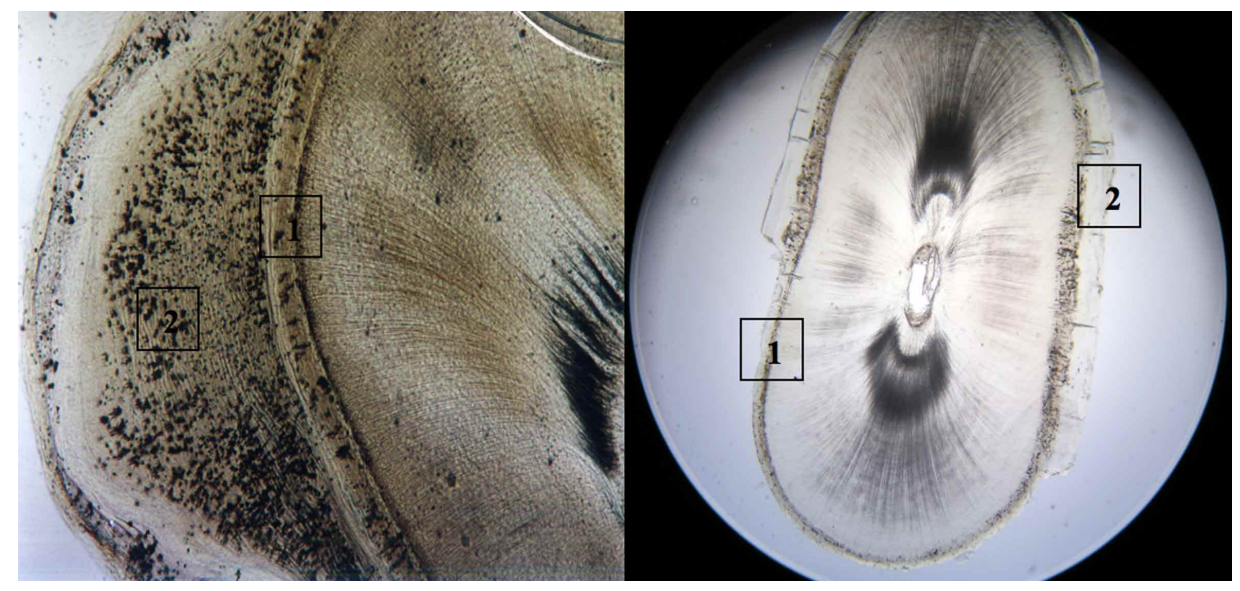

Fig. 2. Visualización de zona granulosa (1) y lagunas (2) en G1 y G2.

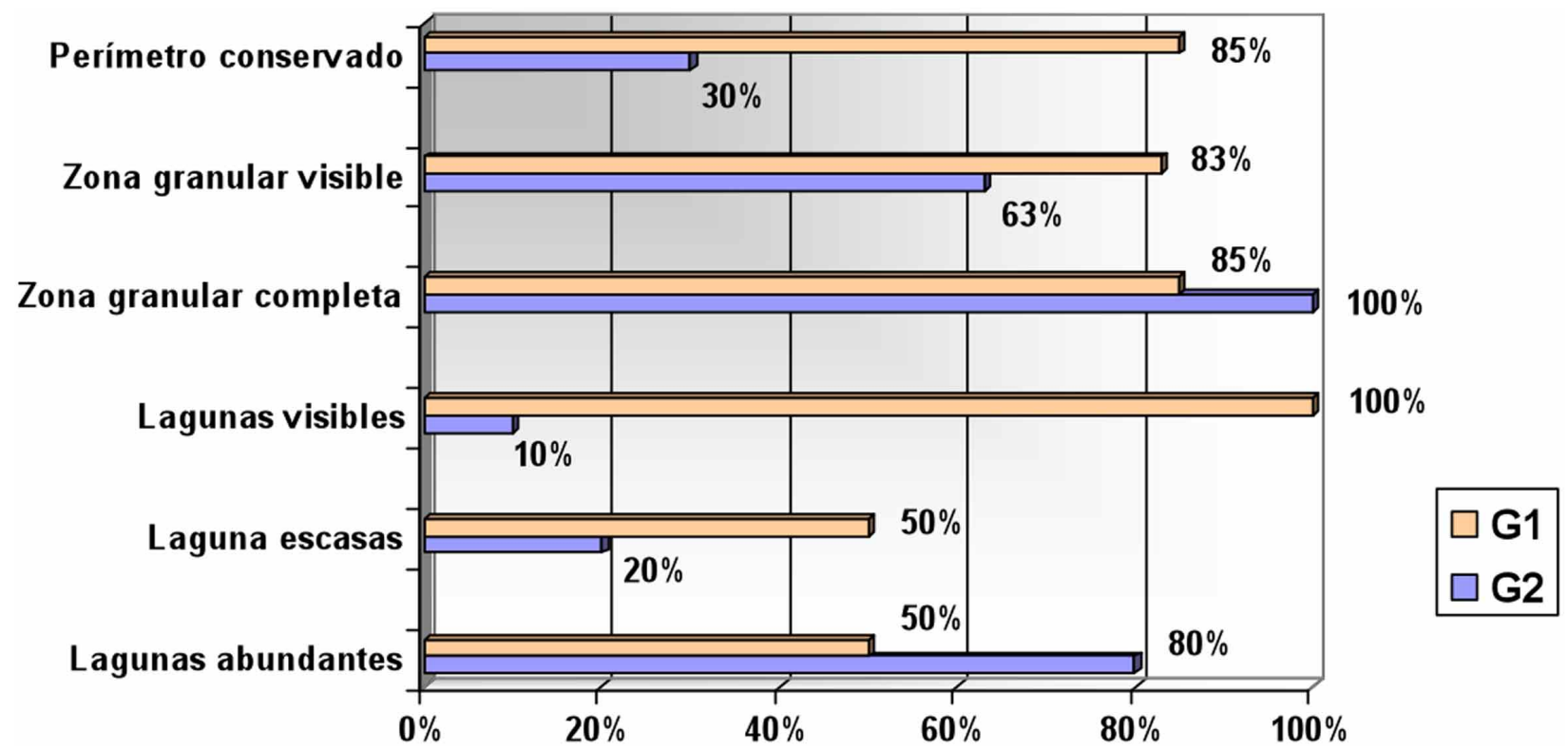

Fig. 3. Distribución de cantidad y visibilidad de lagunas y zona granulosa.
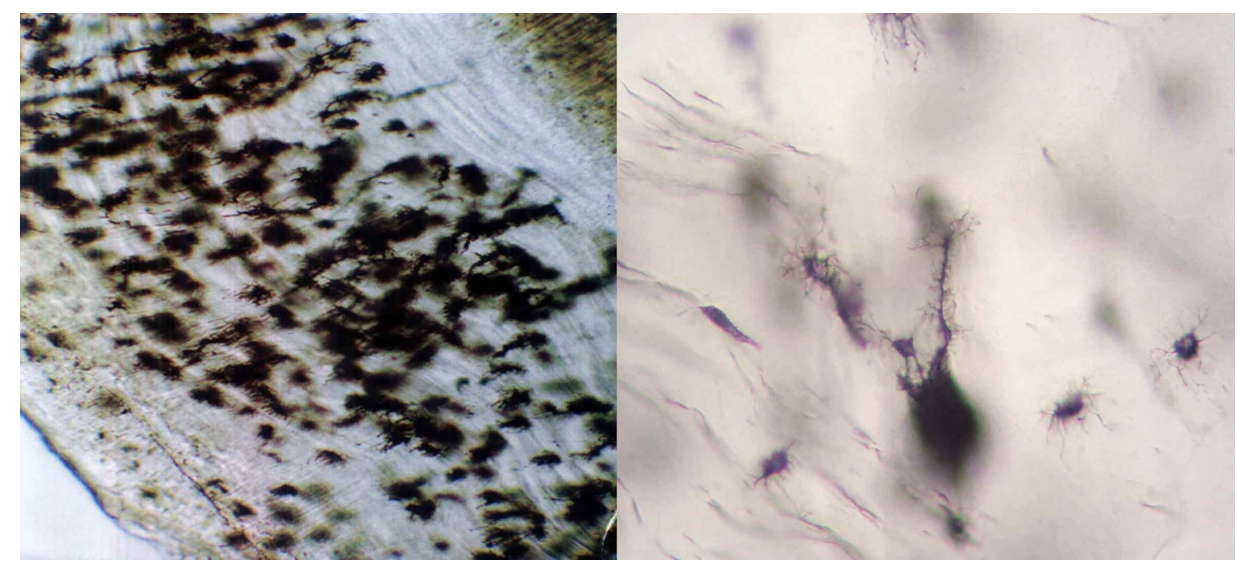

Fig. 4. Lagunas desordenadas a menor y mayor aumento en G2. 


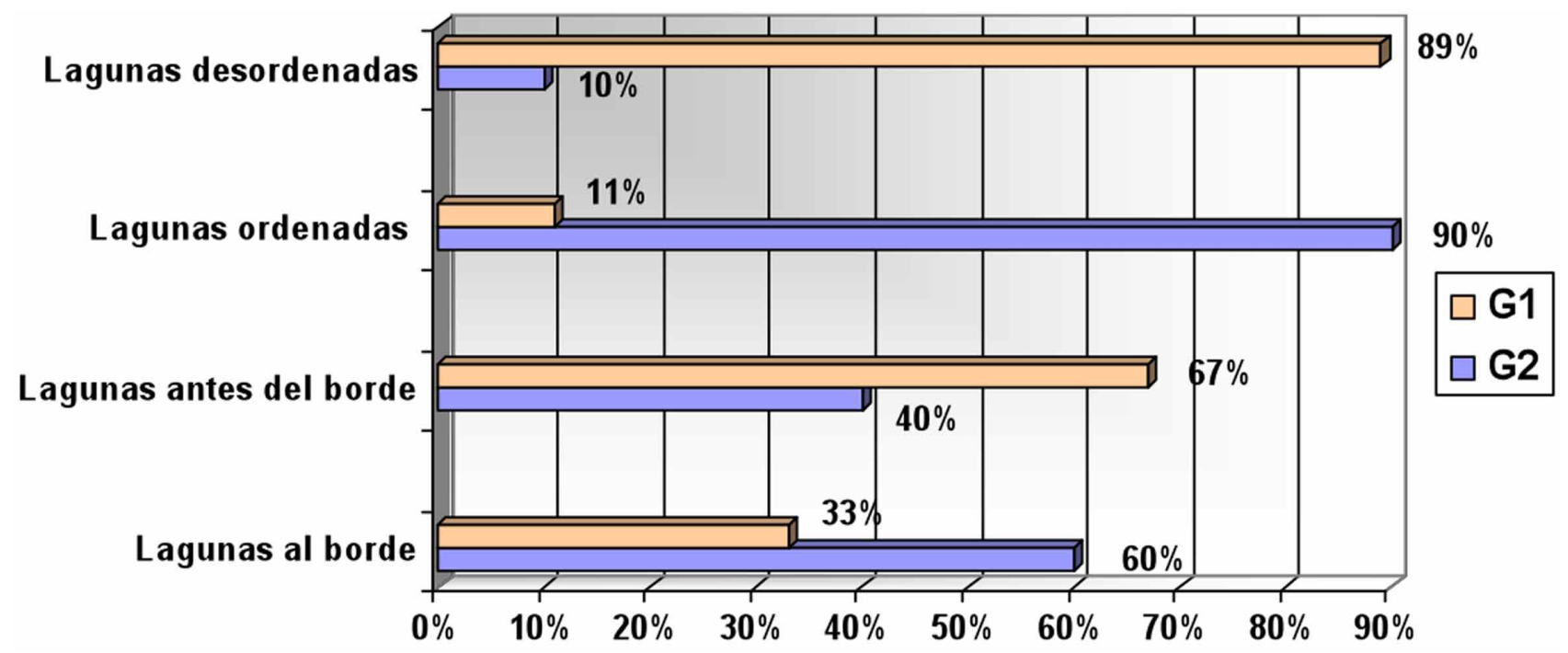

Fig. 5. Distribución y disposición de lagunas en ambos grupos.

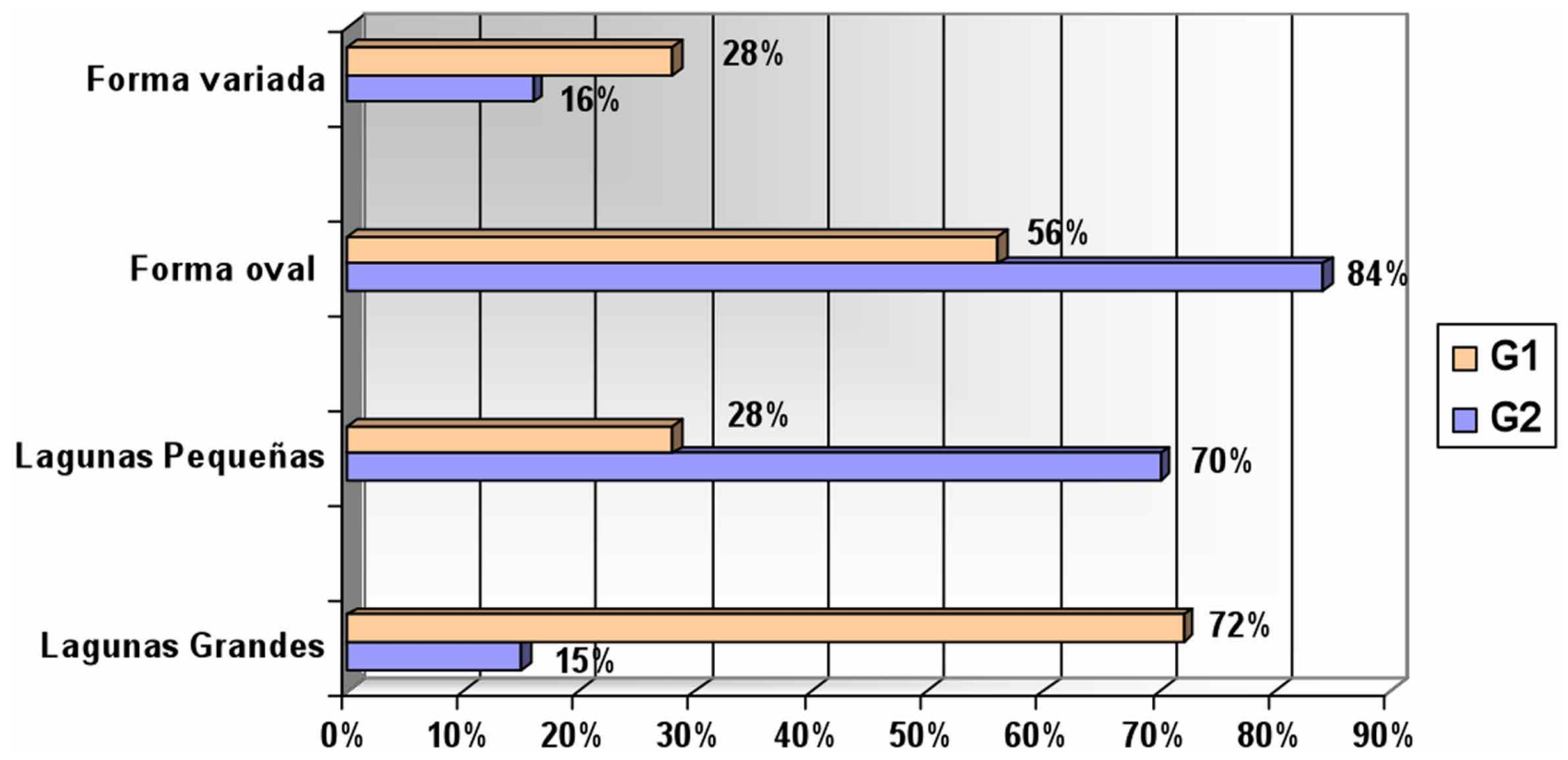

Fig. 6. Formas y tamaños de las lagunas en ambos grupos.

A menor aumento, las lagunas de tamaño grande fueron visibles en el $72 \%$ del G1 y las pequeñas, visibles a mayor aumento, predominaron en el 70\% del G2 ( $\mathrm{p}=0,004)$; su forma, ovales en ambos grupos, se encontraron en menor proporción $55,6 \%$ en G1 y mayor $84 \%$ en G2 (p=0,088); (Fig. 6).

\section{DISCUSIÓN}

En los dientes con raíces normales el contorno fue regular, en el grupo de raíces con lesión las perlas sobresalieron en la superficie del cemento semejante a la deforma- 
ción de una hipercementosis difusa (Cabrini). Los grupos no difirieron en edad ni sexo.

La imagen radiográfica reveló que fueron diferentes en el contorno exterior ya que éste fue liso en la totalidad de las perlas, mientras que en las raíces normales no lo fue por la inserción de fibras periodontales, coincidiendo con descripciones de superficies cementarias normales (Kohli et al., 2011; Abramovich). No encontramos publicaciones científicas referentes a la diferencia entre el cemento normal y la superficie lisa de las perlas, sugerimos que sería consecuencia de la ausencia de inserción fibrilar en dicha zona. El grosor del cemento en las perlas fue mayor en la lesión, diferenciándose del resto de la raíz. Siendo fino en las raíces normales, coincidiendo con la descripción de distintos espesores acorde al tercio de corte de la raíz (Abramovich). Si bien carecemos de bibliografía para comparar nuestros resultados, se observó que el perímetro de la perla se conservó mejor que el de raíces normales, posiblemente por la mineralización demostrada con la radiografía.

La zona granulosa en todo el perímetro fue visible en perlas y dientes normales. La desaparición parcial de la zona granular y el avance dentinario sobre el cemento se observó en menor proporción en el grupo de perlas, sin embargo éstas predominaron en dientes posteriores y nuestro estudio incluyó personas jóvenes. Al no haberse realizado estudios patológicos no podemos asegurar que se trate de un dentinoma (Anker \& Radden, 1989).

En un estudio de hipercementosis utilizando tinción metálica se observaron alteraciones en la forma y tamaño de los cementocitos (Lia et al.). En nuestro trabajo, en los dientes preparados por desgaste, no se observaron células pero se visualizaron los espacios ocupados por ellas llamados lagunas. Estas fueron visibles en la totalidad de las perlas y no lo fueron en la mayoría del cemento normal. Si bien hubo diferencia entre grupos debido a que las lagunas fue- ron más abundantes, desordenadas y llegaron en menor proporción al borde del tejido en las perlas, no hallamostrabajos científicos para comparar nuestros resultados. La forma oval de las lagunas fue predominante en ambos grupos, al igual que lo observado en estudios anteriores (Lia et al). En este trabajo encontramos diferencia en el tamaño de las lagunas, ya que en las perlas predominaron lagunas grandes y en el cemento normal las de tamaño pequeño. Dicho investigador halló cambios de tipo degenerativo representados por la fragmentación de prolongaciones citoplasmáticas de los cementocitos y la existencia de vasos sanguíneos y capilares cerca del límite cemento-dentinario. Utilizando microscopio confocal se estudió a los cementocitos y sus lagunas sugiriendo que estas células podrían tener un rol en la calcificación delcemento secundario (Kagayama et al.). En las perlas no observamos cementocitos incluidos, considerados responsables del mantenimiento del colágeno y de la sustancia fundamental amorfa que luego calcificará, si bien observamos las lagunas ocupadas por dichas células.

Utilizando microscopio electrónico de barrido se evaluaron las características morfológicas de las hipercementosis concluyendo que no estarían relacionadas con la reabsorción considerando que serían un mecanismo de reacción frente a lesiones periapicales crónicas (Pinheiro et al., 2008). Estos hallazgos serían coincidentes con los de nuestro trabajo en el cual el motivo de extracción de las piezas con perlas fue predominantemente la presencia de caries crónica y sus consecuencias apicales, si bien la movilidad dentaria se observó en menor proporción.

En nuestro trabajo se observó el contorno exterior de las perlas liso, conservando su perímetro, la zona granulosa de Tomes más visible al igual que las lagunas, que fueron más abundantes, de mayor tamaño y con distribución desordenada observándose en todas las evaginaciones y llegando en menor proporción al borde del tejido comparado con el cemento normal.

KOHLI, A.; PEZzOTTO, S. M. \& POLETTO, L. Normal human dental roots and with cementum pearls. Structural histological comparison. Int. J. Morphol., 31(3):1020-1025, 2013.

SUMMARY: During tooth life in mouth cellular cement settles in the apex in order to compensate the occlusal wear considered normal. After a tooth extraction, on rare occasions it is possible to visualize cement excesses with pearls form well delimited and hard to tact, located in another radicular sector different of the apex. Our aim was to compare structures in both types of cement in order to find histological differences. Consequently, 20 permanent teeth with pearls (G1) and 20 with normal roots (G2) were used. G1 roots were cut by a thin grain metallic disc at low speed in transverse sense, at the height of the protuberance most prominent part; G2 roots were marked in thirds and cut in transverse sense at the half of the third apical. From both groups a segment was obtained to be reserved and other one that was prepared by erosion technology to be visualized by MO at minor and major increases. Results were compared with student $\mathrm{t}$ test, categorical variables with Fisher's tests significance 5\%. Forty adult patients teeth were obtained, male $42.5 \%$ and female $57.5 \%$, age average in G1 $61 \pm 16$ and $55 \pm 18$ in G2 (p=0.289). Pearls exterior face was smooth, with a preserved perimeter, Tomes granular zone was visible as were lagoons, which were less abundant, greater in size and with uneven distribution arriving at tissue border in a lesser proportion compared with normal cement.

KEY WORDS: Dental cementum; Hypercementosis; Cementum pearls. 


\section{REFERENCIAS BIBLIOGRÁFICAS}

Abramovich, A. Histología y Embriología dentaria. $2^{\mathrm{a}}$ ed. Buenos Aires, Editorial Panamericana, 1999.

Aguiló, L. \& Gandía, J. Evaginación radicular. Rev. Act. Odontoestomat. Esp., 55:7-60, 1995.

Anker, A. H. \& Radden, B. G. Dentinoma of the mandible. Oral Surg. Oral Med. Oral Pathol., 67(6):731-3, 1989.

Avery, J. K. \& Chiego, D. J. Principios de Histología y Embriología bucal con orientación clínica. $3^{\mathrm{a}}$ ed. Buenos Aires, Elsevier, 2007.

Cabrini, R. Anatomía Patológica Bucal. Buenos Aires, Mundi, 1980.

Corruccini, R. S.; Jacobi, K. P.; Handler, J. S. \& Aufderheide, A. C. Implications of tooth root hypercementosis in Barbados slave skeletal collection. Am. J. Phys. Anthropol., 74(2):17984, 1987

Kagayama, M.; Sasano, Y.; Misoguchi, I. \& Takahashi, I. Confocal microscopy of cementocytes and lacunae and canaliculi in rat molars. Anat. Embryol. (Berl.), 195(6):491-6, 1997.

Kohli, A.; Pezzotto, S. M. \& Poletto, L. Hipercementosis apicales y no apicales en raíces dentarias humanas. Int. J. Morphol., 29(4):1263-67, 2011.

Lia, R. C.; Lauand, F.; Marcantonio, E. \& Neto, C. B. A contribution to the histological study of hypercementosis using metal staining. J. Dent. Res., 57(1):146-52, 1978.

Pinheiro, B.; Pinheiro, T; Capelozza, A. \& Consolaro, A. A scanning electron microscopic study of hypercementosis. J. Appl. Oral Sci., 16(6):380-4, 2008.

\section{Dirección para Correspondencia: \\ Alicia Kohli \\ Pasaje Rizzuto 3473 \\ Rosario 2000, Santa Fe \\ ARGENTINA}

Tel: 0341-4612728

Email: Aliciakohli2009@hotmail.com

Recibido : 07-09-2012

Aceptado: 19-05-2013 